\title{
Complete genome sequence of Cryptobacterium curtum type strain $\left(12-3^{\top}\right)$
}

\author{
Konstantinos Mavrommatis ${ }^{1}$, Rüdiger Pukall ${ }^{2}$, Christine Rohde ${ }^{2}$, Feng Chen ${ }^{1}$, David Sims ${ }^{1,3}$, \\ Thomas Brettin $^{1,3}$, Cheryl Kuske ${ }^{1,3}$, John C. Detter ${ }^{1,3}$, Cliff Han ${ }^{1,3}$, Alla Lapidus ${ }^{1}$, Alex Copel- \\ and ${ }^{1}$, Tijana Glavina Del Rio ${ }^{1}$, Matt Nolan ${ }^{1}$, Susan Lucas ${ }^{1}$, Hope Tice ${ }^{1}$, Jan-Fang Cheng ${ }^{1}$, Da- \\ vid Bruce ${ }^{1,3}$, Lynne Goodwin ${ }^{1,3}$, Sam Pitluck ${ }^{1}$, Galina Ovchinnikova ${ }^{1}$, Amrita Pati ${ }^{1}$, Natalia \\ Ivanova $^{1}$, Amy Chen ${ }^{4}$, Krishna Palaniappan ${ }^{4}$, Patrick Chain ${ }^{1,5}$, Patrik D'haeseleer ${ }^{1,5}$, Markus \\ Göker $^{2}$, Jim Bristow ${ }^{1}$, Jonathan A. Eisen ${ }^{1,6}$, Victor Markowitz ${ }^{4}$, Philip Hugenholtz ${ }^{1}$, Manfred \\ Rohde $^{7}$, Hans-Peter Klenk ${ }^{2}$, and Nikos C. Kyrpides ${ }^{1 *}$ \\ ${ }^{1}$ DOE Joint Genome Institute, Walnut Creek, California, USA \\ ${ }^{2}$ DSMZ - German Collection of Microorganisms and Cell Cultures GmbH, Braunschweig, \\ Germany \\ ${ }^{3}$ Los Alamos National Laboratory, Bioscience Division, Los Alamos, New Mexico, USA \\ ${ }^{4}$ Biological Data Management and Technology Center, Lawrence Berkeley National Labora- \\ tory, Berkeley, California, USA \\ ${ }^{5}$ Lawrence Livermore National Laboratory, Livermore, California, USA \\ ${ }^{6}$ University of California Davis Genome Center, Davis, California, USA \\ ${ }^{7} \mathrm{HZI}$ - Helmholtz Centre for Infection Research, Braunschweig, Germany
}

${ }^{*}$ Corresponding author: Nikos C. Kyrpides

Keywords oral infections, opportunistic pathogenic, periodontitis, non-spore-former, anaerobic, asaccharolytic, Coriobacteriaceae

Cryptobacterium curtum Nakazawa et al. 1999 is the type species of the genus, and is of phylogenetic interest because of its very distant and isolated position within the family Coriobacteriaceae. C. curtum is an asaccharolytic, opportunistic pathogen with a typical occurrence in the oral cavity, involved in dental and oral infections like periodontitis, inflammations and abscesses. Here we describe the features of this organism, together with the complete genome sequence, and annotation. This is the first complete genome sequence of the actinobacterial family Coriobacteriaceae, and this 1,617,804 bp long single replicon genome with its 1364 protein-coding and 58 RNA genes is part of the Genomic Encyclopedia of Bacteria and Archaea project.

\section{Introduction}

Strain $12-3^{\mathrm{T}}$ (= DSM $15641=$ ATCC $700683=$ CCUG 43107) is the type strain of Cryptobacterium curtum, which is the sole species within the genus Cryptobacterium [1]. C. curtum was described by Nakazawa et al. in 1999 [1]. The organism is of significant interest because of its position in the tree of life where it was initially wrongly placed close to Eubacterium (Firmicutes) to be then relocated in the phylum Actinobacteria, close to the Coriobacteriaceae [1]. Here we present a summary classification and a set of features for C. curtum $12-3^{\mathrm{T}}$, together with the description of the complete genomic sequencing and annotation.

\section{Classification and features}

The type strain $12-3^{\mathrm{T}}$ and a second strain of the species, KV43-B, both classified in C. curtum were isolated from a periodontal pocket sample of an adult patient and from necrotic dental pulp, respectively [1]. C. curtum can also be isolated from human oral and dental infections like pulpal inflammations, advanced caries [1], dental abscesses or periodontitis [2]. 16S rRNA gene sequence analysis revealed that the two isolates represent a distinct lineage within the family Coriobacteriaceae, between the neighboring genera Eggerthella and Slackia (Figure 1). No significant matches with any 16S rRNA sequences from environmental ge- 
nomic samples and surveys are reported at the NCBI BLAST server (February 2009).

The very short and non-motile rods form tiny translucent colonies of less than $1 \mathrm{~mm}$ in diameter on BHI-blood agar without hemolysis after prolonged incubation under strictly anaerobic conditions (Table 1). Transmission electron micrographs of ultrathin sections of $C$. curtum 12-3 showed a single-layered Gram-positive cell wall of approximately $10 \mathrm{~nm}$ thickness (Figure 2) [1]. Carbohydrates are not metabolized; the species is asaccharolytic [1]. C. curtum is non-reactive in most biochemical tests. The human oral cavity contains arginine and other amino acids and oligopeptides due to proteinase and peptidase activities. C. curtum degrades arginine through arginine deiminase pathway [15]. Like Slackia exigua, a closely related species, these bacteria are very difficult to cultivate. Optimal doubling time is 12 hours [15]. There are no chemotaxonomic data available to $C$. curtium strain $12-3^{\mathrm{T}}$.

Figure 1 shows the phylogenetic neighborhood of $C$. curtum strain $12-3^{\mathrm{T}}$ in a $16 \mathrm{~S}$ rRNA based tree. Analysis of the three 16S rRNA gene sequences in the genome of strain $12-3^{\mathrm{T}}$ indicated that the genes differ by at most one nucleotide from each other, but differ by 15 nucleotides and eight ambiguities $(1.1 \%)$ from the previously published $16 \mathrm{~S}$ rRNA sequence generated from DSM 15641 (AB019260). The higher sequence coverage and overall improved level of sequence quality in whole-genome sequences, as compared to ordinary gene sequences, implies that the significant differences between the genome data and the reported 16S rRNA gene sequence might be due to sequencing errors in the previously reported sequence data.

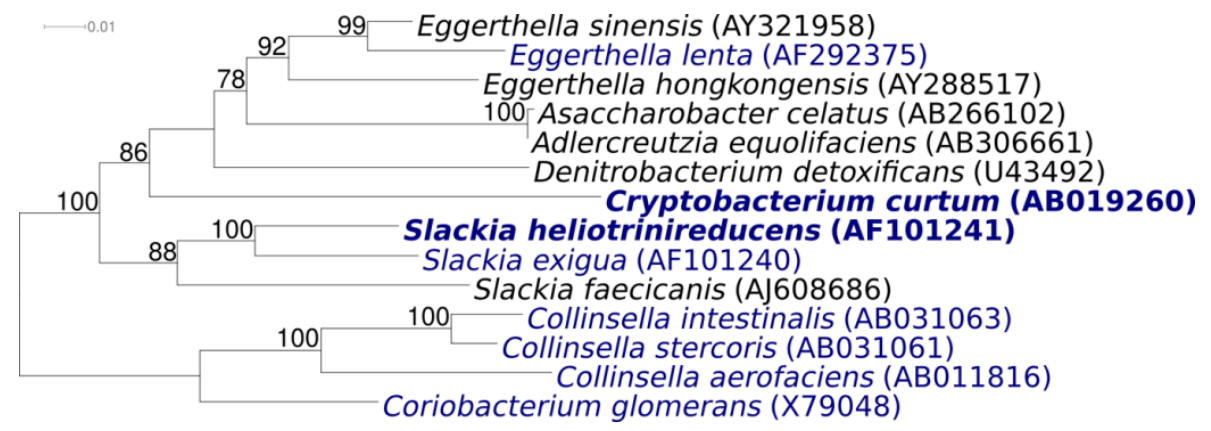

Figure 1. Phylogenetic tree of C. curtum $12-3^{\top}$ and most type strains of the family Coriobacteriaceae, inferred from 1422 aligned 16S rRNA characters [3,4] under the maximum likelihood criterion [5]. The tree was rooted with type strains of the genera Collinsella and Coriobacterium. The branches are scaled in terms of the expected number of substitutions per site. Numbers above branches are support values from 1000 bootstrap replicates if larger than 60\%. Strains with a genome sequencing project registered in GOLD [6] are printed in blue; published genomes in bold, including two of which are reported in this issue of SIGS $[7,8]$

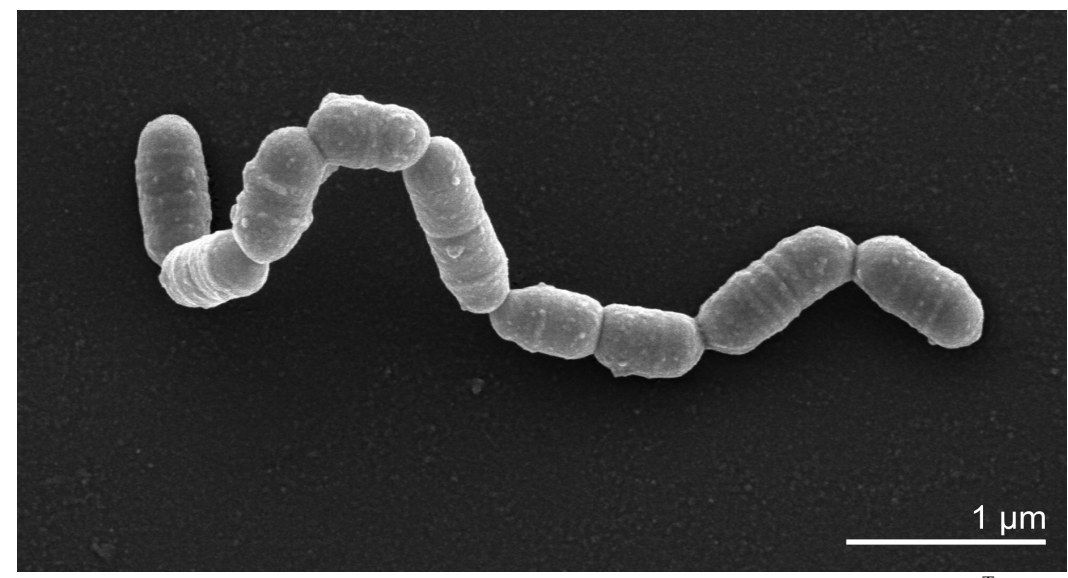

Figure 2. Scanning electron micrograph of C. curtum $12-3^{\top}$ 
Mavrommatis et al.

Table 1. Classification and general features of C. curtum $12-3^{\top}$ according to the MIGS recommendations [9]

\begin{tabular}{|c|c|c|c|}
\hline MIGS ID & Property & Term & $\begin{array}{l}\text { Evidence } \\
\text { code }\end{array}$ \\
\hline & \multirow{8}{*}{ Current classification } & Domain Bacteria & TAS [10] \\
\hline & & Phylum Actinobacteria & TAS [11] \\
\hline & & Class Actinobacteria & TAS [12] \\
\hline & & Order Coriobacteriales & TAS [12] \\
\hline & & Family Coriobacteriaceae & TAS [12] \\
\hline & & Genus Cryptobacterium & TAS $[1]$ \\
\hline & & Species Cryptobacterium curtum & TAS [1] \\
\hline & & Type strain $12-3$ & TAS $[1]$ \\
\hline & Gram stain & positive & TAS [1] \\
\hline & Cell shape & very short rods & TAS [1] \\
\hline & Motility & nonmotile & TAS [1] \\
\hline & Sporulation & non-sporulating & TAS [1] \\
\hline & Temperature range & mesophile & TAS [1] \\
\hline & Optimum temperature & $37^{\circ} \mathrm{C}$ & NAS \\
\hline & Salinity & normal & TAS [1] \\
\hline \multirow[t]{3}{*}{ MIGS-22 } & Oxygen requirement & obligate anaerobic & TAS [1] \\
\hline & Carbon source & asaccharolytic & TAS [1] \\
\hline & Energy source & arginine, lysine & NAS \\
\hline MIGS-6 & Habitat & human oral microflora & TAS $[1]$ \\
\hline MIGS-15 & Biotic relationship & $\begin{array}{l}\text { free living, growth on enzymatic degradation } \\
\text { products of inflamed tissues }\end{array}$ & NAS \\
\hline \multirow[t]{3}{*}{ MIGS-14 } & Pathogenicity & periodontal infections & TAS [1] \\
\hline & Biosafety level & $1(+)$ & TAS $[13]$ \\
\hline & Isolation & infected human oral cavity & TAS [1] \\
\hline MIGS-4 & Geographic location & not reported & NAS \\
\hline MIGS-5 & Sample collection time & about 1995 & TAS [1] \\
\hline MIGS-4.1 & Latitude - Longitude & not reported & \\
\hline MIGS-4.2 & & & \\
\hline MIGS-4.3 & Depth & not reported & \\
\hline MIGS-4.4 & Altitude & not reported & \\
\hline
\end{tabular}

Evidence codes - IDA: Inferred from Direct Assay (first time in publication); TAS: Traceable Author Statement (i.e., a direct report exists in the literature); NAS: Non-traceable Author Statement (i.e., not directly observed for the living, isolated sample, but based on a generally accepted property for the species, or anecdotal evidence). These evidence codes are from the Gene Ontology project [14]. If the evidence code is IDA, then the property was directly observed for a live isolate by one of the authors, or an expert or reputable institution mentioned in the acknowledgements.

\section{Genome sequencing and annotation Genome project history}

This organism was selected for sequencing on the basis of each phylogenetic position, and is part of the Genomic Encyclopedia of Bacteria and Archaea project. The genome project is deposited in the Genome OnLine Database [6] and the complete genome sequence in GenBank. Sequencing, finishing and annotation were performed by the DOE Joint Genome Institute (JGI). A summary of the project information is shown in Table 2.

\section{Growth conditions and DNA isolation}

C. curtum strain 12-3 ${ }^{\mathrm{T}}$, DSM 15641, was grown anaerobically in DSMZ medium 78 (Chopped Meat Medium) [16], supplemented with $1 \mathrm{~g} / \mathrm{l}$ arginine, at $37^{\circ} \mathrm{C}$. DNA was isolated from 1-1.5 g of cell paste using Qiagen Genomic 500 DNA Kit (Qiagen, Hilden, Germany) with protocol modification st/FT [17] for cell lysis. 


\section{Genome sequencing and assembly}

The genome was sequenced using a combination of Sanger and 454 sequencing platforms. All general aspects of library construction and sequencing performed at the JGI can be found at http://www.jgi.doe.gov/. 454 Pyrosequencing reads were assembled using the Newbler assembler version 1.1.02.15 (Roche). Large Newbler contigs were broken into 1,799 overlapping fragments of $1000 \mathrm{bp}$ and entered into assembly as pseudo-reads. The sequences were assigned quality scores based on Newbler consensus q-scores with modifications to account for overlap redun- dancy and to adjust inflated q-scores. A hybrid 454/Sanger assembly was made using the parallel phrap assembler (High Performance Software, LLC). Possible mis-assemblies were corrected with Dupfinisher [18] or transposon bombing of bridging clones (Epicentre Biotechnologies, Madison, WI). Gaps between contigs were closed by editing in Consed, custom primer walk or PCR amplification. 47 Sanger finishing reads were produced to close gaps, to resolve repetitive regions, and to raise the quality of the finished sequence. The error rate of the completed genome sequence is less than 1 in 100,000 . Together all sequence types provided $32.9 x$ coverage of the genome.

Table 2. Genome sequencing project information

\begin{tabular}{|c|c|c|}
\hline MIGS ID & Property & Term \\
\hline MIGS-31 & Finishing quality & Finished \\
\hline MIGS-28 & Libraries used & $\begin{array}{l}\text { Three genomic libraries: two Sanger li- } \\
\text { braries - } 8 \text { kb pMCL200 and fosmid } \\
\text { pcc1Fos - and } \\
\text { one } 454 \text { pyrosequence standard library }\end{array}$ \\
\hline MIGS-29 & Sequencing platforms & $\mathrm{ABI} 3730,454 \mathrm{GS} F L X$ \\
\hline MIGS-31.2 & Sequencing coverage & $12.9 \times$ Sanger; $20 \times$ pyrosequence \\
\hline MIGS-30 & Assemblers & Newbler version 1.1.02.15, phrap \\
\hline \multirow[t]{6}{*}{ MIGS-32 } & Gene calling method & $\begin{array}{l}\text { Genemark 4.6b, tRNAScan-SE-1.23, in- } \\
\text { fernal } 0.81 \text {, GenePRIMP }\end{array}$ \\
\hline & INSDC / Genbank ID & СР001682 \\
\hline & Genbank Date of Release & August 26, 2009 \\
\hline & GOLD ID & Gc01086 \\
\hline & NCBI Project ID & 20739 \\
\hline & Database: IMG-GEBA & 2500901758 \\
\hline \multirow[t]{2}{*}{ MIGS-13 } & Source material identifier & DSM 15641 \\
\hline & Project relevance & Tree of Life, GEBA \\
\hline
\end{tabular}

\section{Genome annotation}

Genes were identified using GeneMark [19] as part of the genome annotation pipeline in the Integrated Microbial Genomes Expert Review (IMG-ER) system [20], followed by a round of manual curation using the JGI GenePRIMP pipeline [21]. The predicted CDSs were translated and used to search the National Center for Biotechnology Information (NCBI) nonredundant database, UniProt, TIGRFam, Pfam, PRIAM, KEGG, COG, and InterPro databases. The tRNAScanSE tool [22] was used to find tRNA genes, whereas ribosomal RNAs were found by using the tool RNAmmer [23]. Other non coding RNAs were identified by searching the genome for the Rfam profiles using INFERNAL (v0.81) [24]. Additional gene prediction analysis and manual functional annotation was performed within the Integrated Microbial Genomes (IMG) platform (http://img.jgi.doe.gov) [25].

\section{Metabolic network analysis}

The metabolic Pathway/Genome Database (PGDB) was computationally generated using Pathway Tools software version 12.5 [26] and MetaCyc version 12.5 [27], based on annotated EC numbers and a customized enzyme name mapping file. It has undergone no subsequent manual curation and may contain errors, similar to a Tier 3 BioCyc PGDB [28].

\section{Genome properties}

The genome is $1,617,804 \mathrm{bp}$ long and comprises one main circular chromosome with a $50.9 \%$ GC content (Table 3 and Figure 3). Of the 1422 genes 
predicted, 1364 were protein coding genes, and 58 RNAs. A total of 7 pseudogenes were also identified. Among the majority of protein coding genes $(78.5 \%)$ were assigned with a putative function while the remaining were annotated as hypothetical proteins. The properties and the statistics of the genome are summarized in Table 3. The distri- bution of genes into COG functional categories is presented in Table 4, and a cellular overview diagram is presented in Figure 4, followed by a summary of metabolic network statistics shown in Table 5 .

Table 3. Genome Statistics

\begin{tabular}{lrr}
\hline Attribute & Value & \% of Total \\
\hline Genome size (bp) & $1,617,804$ & \\
DNA Coding region (bp) & $1,439,290$ & $88.97 \%$ \\
DNA G+C content (bp) & 823,649 & $50.91 \%$ \\
Number of replicons & 1 & \\
Extrachromosomal elements & 0 & \\
Total genes & 1425 & $100.00 \%$ \\
RNA genes & 58 & $2.37 \%$ \\
rRNA operons & 3 & \\
Protein-coding genes & 1364 & $95.92 \%$ \\
Pseudo genes & 7 & $0.49 \%$ \\
Genes with function prediction & 1117 & $78.55 \%$ \\
Genes in paralog clusters & 77 & $5.41 \%$ \\
Genes assigned to COGs & 1103 & $77.57 \%$ \\
Genes assigned Pfam domains & 1104 & $77.64 \%$ \\
Genes with signal peptides & 276 & $19.37 \%$ \\
Genes with transmembrane helices & 206 & $14.46 \%$ \\
CRISPR repeats & 0 & \\
\hline
\end{tabular}

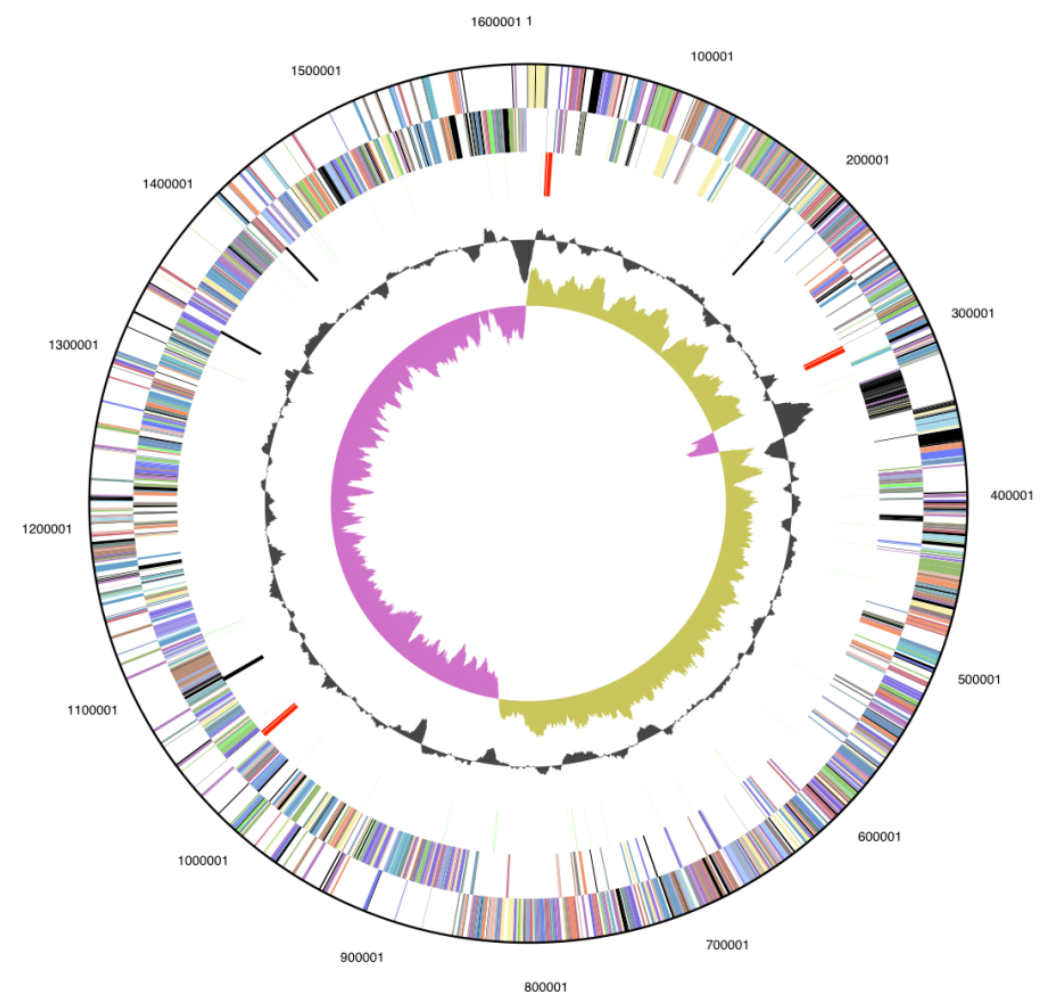

Figure 3. Graphical circular map of the genome. From outside to the center: Genes on forward strand (color by COG categories), Genes on reverse strand (color by COG categories), RNA genes (tRNAs green, rRNAs red, other RNAs black), GC content, GC skew. 
Table 4. Number of genes associated with the general COG functional categories

\begin{tabular}{lrrl}
\hline Code & Value & \multicolumn{1}{c}{$\%$} & Description \\
\hline J & 128 & 9.4 & Translation, ribosomal structure and biogenesis \\
A & 1 & 0.1 & RNA processing and modification \\
K & 94 & 6.9 & Transcription \\
L & 74 & 5.5 & Replication, recombination and repair \\
B & 1 & 0.1 & Chromatin structure and dynamics \\
D & 15 & 1.1 & Cell cycle control, mitosis and meiosis \\
Y & 0 & 0.0 & Nuclear structure \\
V & 20 & 1.5 & Defense mechanisms \\
T & 64 & 4.7 & Signal transduction mechanisms \\
M & 70 & 5.1 & Cell wall/membrane biogenesis \\
N & 1 & 0.1 & Cell motility \\
Z & 1 & 0.1 & Cytoskeleton \\
W & 0 & 0.0 & Extracellular structures \\
U & 20 & 1.5 & Intracellular trafficking and secretion \\
O & 55 & 4.0 & Posttranslational modification, protein turnover, chaperones \\
C & 100 & 7.3 & Energy production and conversion \\
G & 41 & 3.0 & Carbohydrate transport and metabolism \\
E & 96 & 7.0 & Amino acid transport and metabolism \\
F & 47 & 3.4 & Nucleotide transport and metabolism \\
H & 69 & 5.1 & Coenzyme transport and metabolism \\
I & 39 & 2.9 & Lipid transport and metabolism \\
P & 70 & 5.1 & Inorganic ion transport and metabolism \\
Q & 9 & 0.7 & Secondary metabolites biosynthesis, transport and catabolism \\
R & 119 & 8.7 & General function prediction only \\
S & 81 & 5.9 & Function unknown \\
- & 261 & 19.1 & Not in COGs \\
\hline
\end{tabular}

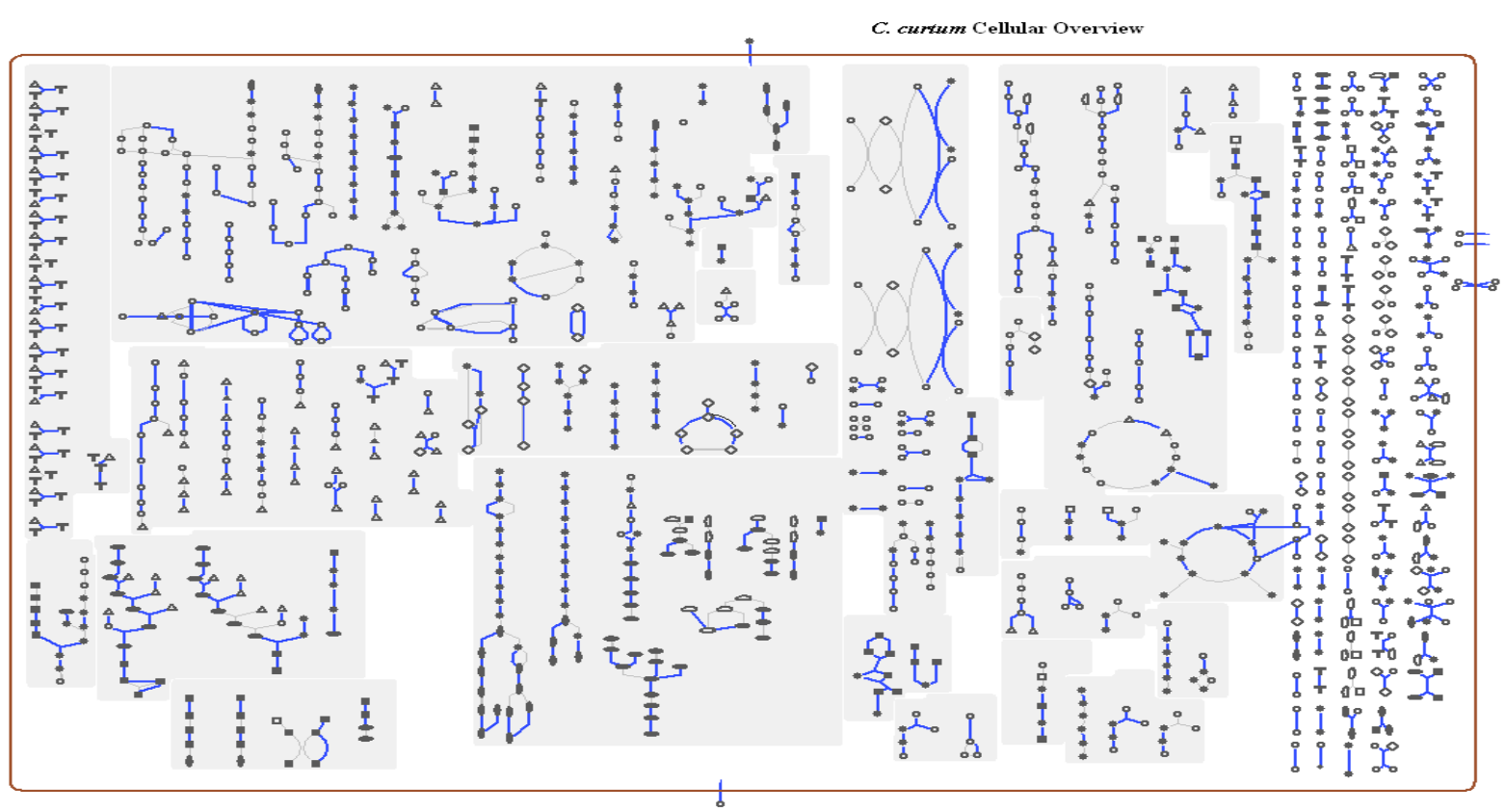

Figure 4. Schematic cellular overview diagram of all pathways of $C$. curtum $12-3^{\top}$. Nodes represent metabolites, with shape indicating class of metabolite. Lines represent reactions. 
Table 5. Metabolic Network Statistics

\begin{tabular}{lr}
\hline Attribute & Value \\
\hline Total genes & 1422 \\
Enzymes & 316 \\
Enzymatic reactions & 606 \\
Metabolic pathways & 115 \\
Metabolites & 506 \\
\hline
\end{tabular}

\section{Acknowledgements}

We would like to gratefully acknowledge the help of Gabriele Gehrich-Schröter for growing C. curtum cultures and Susanne Schneider for DNA extraction and quality analysis (both at DSMZ). This work was performed under the auspices of the US Department of Energy Office of Science, Biological and Environmental Research Program, and by the University of California,

\section{References}

1. Nakazawa F, Poco SE, Ikeda T, Sato M, Kalfas S, Sundqvist G, Hoshino E. Cryptobacterium curtum gen. nov., sp. nov., a new genus of Gram-positive anaerobic rod isolated from human oral cavities. Int J Syst Bacteriol 1999; 49:1193-1200. PubMed

2. Kumar PS, Griffen AL, Barton JA, Paster BJ, Moeschberger ML, Leys EJ. New bacterial species associated with chronic periodontitis. J Dent Res 2003; 82:338-344

PubMed doi:10.1177/154405910308200503

3. Lee C, Grasso C, Sharlow MF. Multiple sequence alignment using partial order graphs. BioinformatiCs 2002; 18:452-464.

PubMed doi:10.1093/bioinformatics/18.3.452

4. Castresana J. Selection of conserved blocks from multiple alignments for their use in phylogenetic analysis. Mol Biol Evol 2000; 17:540-552. PubMed

5. Stamatakis A, Hoover P, Rougemont J. A rapid bootstrap algorithm for the RAxML web-servers. Syst Biol 2008; 57:758-771. PubMed doi:10.1080/10635150802429642

6. Liolios K, Mavromatis K, Tavernarakis N, Kyrpides NC. The Genomes OnLine Database (GOLD) in 2007: status of genomic and metagenomic projects and their associated metadata. Nucleic Acids Res 2008; 36:D475-D479.

PubMed doi:10.1093/nar/gkm884

7. Copeland A, Sikorski J, Lapidus A, Nolan M, Galvina Del Rio T, Lucas S, Chen F, Tice H, Pitluck S, Cheng JF, et al. Complete genome sequence of Atopobium parvulum type strain (IPP 1246T). Stand Genomic Sci 2009; 1: 166173. doi:10.4056/sigs. 15195
Lawrence Berkeley National Laboratory under contract No. DE-AC02-05CH11231, Lawrence Livermore National Laboratory under Contract No. DE-AC5207NA27344, and Los Alamos National Laboratory under contract No. DE-AC02-06NA25396, as well as German Research Foundation (DFG) INST 599/1-1

8. Saunders E, Pukall R, Abt B, Lapidus A, Galvina Del Rio T, Copeland A, Tice H, Cheng JF, Lucas S, Chen $\mathrm{F}$, et al. Complete genome sequence of $\mathrm{Eg}$ gerthella lenta type strain (IPP VPI 0255T). Stand Genomic Sci 2009; 1: 174-

182. doi:10.4056/sigs.33592

9. Field D, Garrity G, Gray T, Morrison N, Selengut J, Sterk P, Tatusova T, Thomson N, Allen MJ, Angiuoli SV, et al. Towards a richer description of our complete collection of genomes and metagenomes: the "Minimum Information about a Genome Sequence" (MIGS) specification. Nat Biotechnol 2008; 26:541-547.

PubMed doi:10.1038/nbt1360

10. Woese CR, Kandler O, Wheelis ML. Towards a natural system of organisms: proposal for the domains Archaea, Bacteria, and Eucarya. Proc Natl Acad Sci USA 1990; 87: 4576-4579. PubMed doi:10.1073/pnas.87.12.4576

11. Garrity GM, Holt J. Taxonomic Outline of the Archaea and Bacteria. Bergey's Manual of Systematic Bacteriology, $2^{\text {nd }}$ Ed. In: Garrity GM, Boone DR, Castenholz RW Eds. Vol 1 The Archaea, Deeply Branching and Phototrophic Bacteria. 2001 pp. $155-166$

12. Stackebrandt E, Rainey FA, Ward-Rainey NL. Proposal for a new hierarchic classification system, Actinobacteria classis nov. Int I Syst Bacteriol 1997; 47:479-491.

13. Anonymous Biological Agents. Technical rules for biological agents www.baua.de TRBA 466.

14. Ashburner M, Ball CA, Blake JA, Botstein D, Butler H, Cherry JM, Davis AP, Dolinski K, Dwight SS, Eppig JT, et al. Gene ontology: tool for the unifi- 
cation of biology. Nat Genet 2000; 25:25-29. PubMed doi:10.1038/75556

15. Uematsu H, Sato N, Djais A, Hoshino E. Degradation of arginine by Slackia exigua ATCC 700122 and Cryptobacterium curtum ATCC 700683. Oral Microbiol Immunol 2006; 21:381-384. PubMed doi:10.1111/j.1399-302X.2006.00307.x

16. Holdeman LV, Cato EP, Moore WEC. Anaerobe Laboratory Manual, $4^{\text {th }}$ Edition. VPI, Virginia, 1977.

17. Wu D, Hugenholtz P, Mavromatis K, Pukall R, Dalin E, Ivanova NN, Kunin V, Goodwin L, Wu $M$, Tindall B et al. A phylogeny-driven genomic encyclopedia of Bacteria and Archaea. Nature, (In press)

18. Han CS, Chain P. Finishing repeat regions automatically with Dupfinisher. In: Proceeding of the 2006 international conference on bioinformatics \& computational biology. Hamid R. Arabnia \& Homayoun Valafar (eds), CSREA Press. June 2629, 2006:141-146.

19. Besemer J, Lomsadze A, Borodovsky M. GeneMarkS: a self-training method for prediction of gene starts in microbial genomes. Implications for finding sequence motifs in regulatory regions. Nucleic Acids Res 2001; 29:2607-2618 $\underline{\text { PubMed doi:10.1093/nar/29.12.2607 }}$

20. Markowitz VM, Mavromatis K, Ivanova NN, Chen IMA, Chu K, Kyrpides NC. Expert Review of Functional Annotations for Microbial Genomes. Bioinformatics 2009;25:2271-2278 PubMed doi:10.1093/bioinformatics/btp393

21. Pati A, Ivanova N, Mikhailova, N, Ovchinikova G, Hooper SD, Lykidis A, Kyrpides NC GenePRIMP: A Gene Prediction Improvement Pipeline for microbial genomes. (submitted)
22. Lowe TM, Eddy SR. tRNAscan-SE: a program for improved detection of transfer RNA genes in genomic sequence. Nucleic Acids Res 1997; 25:955-964 PubMed doi:10.1093/nar/25.5.955

23. Lagesen K, Hallin P, Rødland EA, Staerfeldt $\mathrm{HH}$, Rognes T, Ussery DW. RNAmmer: consistent and rapid annotation of ribosomal RNA genes. Nucleic Acids Res 2007; 35:3100-3108. PubMed doi:10.1093/nar/gkm160

24. Griffiths-Jones S, Moxon S, Marshall M, Khanna A, Eddy SR, Bateman A. Rfam: annotating noncoding RNAs in complete genomes. Nucleic Acids Res 2005; 33:D121-D124. PubMed doi:10.1093/nar/gki081

25. Markowitz VM, Szeto E, Palaniappan K, Grechkin Y, Chu K, Chen IMA, Dubchak I, Anderson I, Lykidis A, Mavromatis K, et al. The Integrated Microbial Genomes (IMG) system in 2007: data content and analysis tool extensions. Nucleic Acids Res 2008; 36:D528-D533. PubMed doi:10.1093/nar/gkm846

26. Karp PD, Paley SM, Romero P. The Pathway Tools Software. Bioinformatics 2002; 18:S225S232. PubMed

27. Karp P, Caspi R, Foerster H, Fulcher CA, Kaipa $P$, Krummenacker M, Latendresse M, Paley SM, Rhee SY, Shearer A, et al. The MetaCyc Database of metabolic pathways and enzymes and the BioCyc collection of pathway/Genome Databases. Nucleic Acids Res 2008; 36:D623-D631. PubMed

28. Karp PD, Ouzounis CA, Moore-Kochlacs C, Goldovsky L, Kaipa P, Ahren D, Tsoka S, Darzentas N, Kunin V, Lopez-Bigas N. Expansion of the BioCyc collection of pathway/genome databases to 160 genomes. Nucleic Acids Res 2005; 33:6083-6089. PubMed doi:10.1093/nar/gki892 